\title{
Impact of Early Intravenous Epinephrine Administration on Outcomes Following Out-of-Hospital Cardiac Arrest
}

\author{
Yasuyuki Hayashi, MD, PhD; Taku Iwami, MD, PhD; Tetsuhisa Kitamura, MD; \\ Tatsuya Nishiuchi, MD, PhD; Kentaro Kajino, MD, PhD; Tomohiko Sakai, MD, PhD; \\ Chika Nishiyama, PhD; Masahiko Nitta, MD, PhD; Atsushi Hiraide, MD, PhD; Tatsuro Kai, MD
}

\begin{abstract}
Background: The effectiveness of epinephrine administration for cardiac arrests has been shown in animal models, but the clinical effect is still controversial.

Methods and Results: A prospective, population-based, observational study in Osaka involved consecutive outof-hospital cardiac arrest (OHCA) patients from January 2007 through December 2009. We evaluated the outcomes among adult non-traumatic bystander-witnessed OHCA patients for whom the local protocol directed the emergency medical service personnel to administer epinephrine. After stratifying by first documented cardiac rhythm, outcomes were compared among the following groups: non-administration, $\leq 10,11-20$ and $\geq 21$ min as the time from emergency call to epinephrine administration. A total of 3,161 patients were eligible for our analyses, among whom $1,013(32.0 \%)$ actually received epinephrine. The epinephrine group had a significantly lower rate of neurologically intact 1-month survival than the non-epinephrine group (4.1\% vs. $6.1 \%, P=0.028)$. In cases of ventricular fibrillation (VF) arrest, patients in the early epinephrine group who received epinephrine administration within $10 \mathrm{~min}$ had a significantly higher rate of neurologically intact 1-month survival compared with the non-epinephrine group $(66.7 \%$ vs. $24.9 \%$ ), though other epinephrine groups did not. In cases of non-VF arrest, the rate of neurologically intact 1 month survival was low, irrespective of epinephrine administration.
\end{abstract}

Conclusions: The effectiveness of epinephrine after OHCA depends on the time of administration. When epinephrine is administered in the early phase, there is an improvement in neurological outcome from OHCA with VF. (Circ $J$ 2012; 76: 1639-1645)

Key Words: Cardiac arrest; Cardiopulmonary resuscitation; Epidemiology; Epinephrine; Sudden death

$\mathbf{S}$ udden cardiac arrest is one of the most important public health problems in developed countries, ${ }^{1}$ and approximately 60,000 cases occur every year in Japan. ${ }^{2}$ To improve survival from out-of-hospital cardiac arrest (OHCA), it is also important to improve the "chain of survival", 3 in which epinephrine administration is a major component of advanced life support (ALS) measures. ${ }^{1}$

Although epinephrine is one of the most widely used resuscitation drugs across the world, its benefits and risks remain controversial. ${ }^{4-8}$ Recently, a major randomized controlled trial (RCT), which assessed survival from OHCA with and without drug administration including epinephrine in the prehospital setting, failed to show improved survival by drug administra- tion, and concluded that it should not be recommended before hospital arrival. ${ }^{9}$

Interestingly, animal studies have indicated that epinephrine improves survival after cardiac arrests. ${ }^{10-13}$ Differences in epinephrine's effects between human and animal studies might be explained by the time difference in epinephrine administration, because the mean time to administer epinephrine in animal studies has been $10 \mathrm{~min}$, against $20 \mathrm{~min}$ in human studies. ${ }^{14-16}$ Therefore, because the effectiveness of epinephrine might be time-dependent, early administration might improve survival in clinical settings, though no studies have been conducted to evaluate the time-dependent effectiveness of epinephrine for OHCA patients. In addition, some studies have

Received December 7, 2011; revised manuscript received February 23, 2012; accepted March 8, 2012; released online April 5, 2012 Time for primary review: 19 days

Senri Critical Care Medical Center, Osaka Saiseikai Senri Hospital, Suita (Y.H., T. Kai); Kyoto University Health Service, Kyoto (T.I., T. Kitamura, C.N.); Department of Critical Care \& Emergency Medicine, Osaka City University Graduate School of Medicine, Osaka (T.N.); Emergency and Critical Care Medical Center, Osaka Police Hospital, Osaka (K.K.); Department of Emergency Medicine, Osaka Medical College, Takatsuki, Japan (M.N.); Department of Acute Medicine, Kinki University Faculty of Medicine, Osaka-Sayama (A.H.); and Department of Trauma and Critical Care Medicine and Burn Centers, Social Insurance Hospital, Nagoya (T.S.), Japan

Mailing address: Yasuyuki Hayashi, MD, PhD, Senri Critical Care Medical Center, Osaka Saiseikai Senri Hospital, 1-1-6 Tsukumodai, Suita 565-0862, Japan. E-mail: yhayashi@ senri.saiseikai.or.jp

ISSN-1346-9843 doi:10.1253/circj.CJ-11-1433

All rights are reserved to the Japanese Circulation Society. For permissions, please e-mail: cj@j-circ.or.jp 


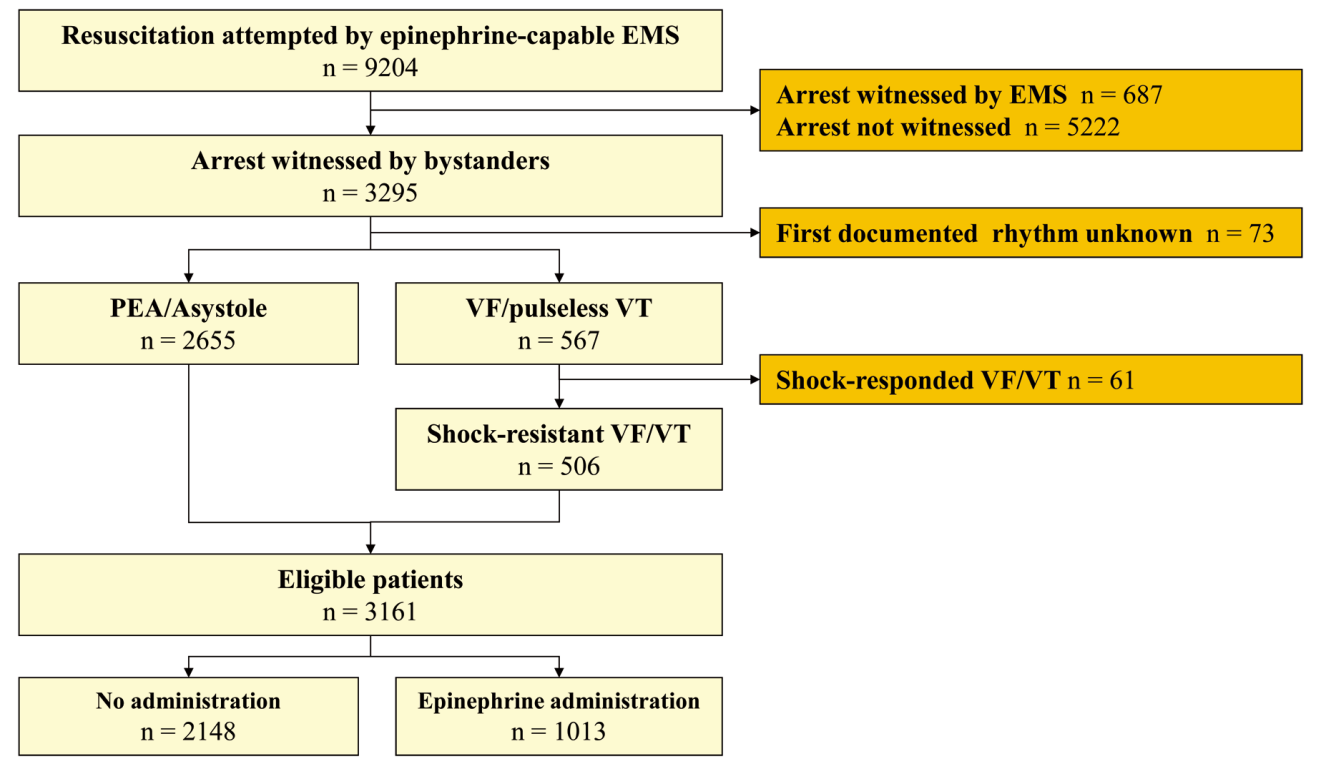

Figure 1. Overview of epinephrine-capable EMS-treated cardiac arrests with an abridged Utstein template from January 1, 2007 through December 31, 2009. EMS, emergency medical service; VF, ventricular fibrillation; VT, ventricular tachycardia; PEA, pulseless electrical activity.

suggested that survival after epinephrine administration would differ depending on the cardiac rhythm (ventricular fibrillation [VF] or non-VF rhythm). 4,9

In Japan, since July 2006, intravenous epinephrine administration by specially trained emergency life saving technicians (ELSTs) under strict medical directions has been legally permitted. The Utstein Osaka Project is a large prospective population-based cohort study of OHCA in Osaka, Japan, covering approximately 8.8 million inhabitants. ${ }^{17,18}$ Using this database, we collected over 3,000 cases of adult non-traumatic bystanderwitnessed OHCA patients who were considered for treatment with epinephrine administration under medical direction (either shock-resistant VF or witnessed arrest with pulseless electrical activity [PEA]/asystole). We then examined the effects of epinephrine on neurological outcomes following OHCA, considering the time to epinephrine administration and the first documented cardiac rhythm. We hypothesized that early intravenous epinephrine administration within $10 \mathrm{~min}$ after OHCA occurrence would improve neurological outcomes compared with non-epinephrine administration, and that the effect would differ by the type of first documented cardiac rhythm.

\section{Methods}

\section{Study Design and Setting}

The Osaka Utstein registry is a large-scale, prospective, population-based registry of OHCA based on the standardized Utstein style. ${ }^{19,20}$ In this observational study, we enrolled all patients aged $\geq 18$ years who had experienced OHCA before the arrival of the emergency medical services (EMS), had been resuscitated by EMS personnel, and then transported to a medical institution in Osaka Prefecture between January 1, 2007 and December 31, 2009. The research protocol was approved by the institutional review board of Osaka University with the assent of the EMS authorities of the local governments in Osaka Prefecture.
Cardiac arrest was defined as the cessation of cardiac mechanical activity, as confirmed by the loss of signs of circulation. ${ }^{19,20}$ An arrest was presumed to be of cardiac etiology unless it was caused by trauma, drowning, drug overdose, asphyxia, exsanguination, or any other noncardiac cause. These diagnoses were determined by the physician in charge in collaboration with the EMS rescuers.

\section{EMS System in Osaka}

Details of the EMS system in Osaka Prefecture have beendescribed previously. ${ }^{17}$ Osaka Prefecture has approximately 8.8 million residents in urban and rural areas totaling $1,892 \mathrm{~km}^{2}$. As of 2011, there were 35 fire stations with a dispatch center, and their EMS systems are essentially all the same. Each ambulance has 3 emergency providers, including at least 1 ELST, who is a highly trained prehospital emergency care provider. ELSTs are allowed to place an intravenous line, insert an adjunct airway, and use semi-automated external defibrillators for OHCA. ${ }^{17}$ Public-access automated external defibrillators were introduced after July, 2004. All EMS providers performed cardiopulmonary resuscitation (CPR) according to the Japanese CPR guidelines based on other national CPR guidelines. ${ }^{1,21-23}$ EMS providers were not permitted to terminate resuscitation in the field.

\section{Study Patients}

Among the non-traumatic bystander-witnessed OHCA patients aged $\geq 18$ years who were resuscitated by epinephrinecapable EMS personnel, those who were eligible for treatment with epinephrine under medical direction (either shock-resistant VF or witnessed arrest with PEA/asystole) were included in these analyses.

\section{Epinephrine Administration by EMS Personnel}

In Japan since July 2006, specially trained ELSTs have been legally allowed to administer intravenous epinephrine in the 


\begin{tabular}{|lrrrr|}
\hline \multicolumn{4}{ll}{ Table 1. Clinical and EMS Characteristics of Eligible Patients With and Without Epinephrine Administration } \\
& Total & $\begin{array}{c}\text { No } \\
\text { administration }\end{array}$ & $\begin{array}{c}\text { Epinephrine } \\
\text { administration }\end{array}$ & P value \\
$\mathrm{n}$ & 3,161 & 2,148 & 1,013 & \\
Age, years, mean (SD) & $73.3(15.2)$ & $73.9(15.2)$ & $72.1(15.0)$ & 0.002 \\
Men, $\mathrm{n}(\%)$ & $1,903(60.2)$ & $1,243(57.9)$ & $660(65.2)$ & $<0.001$ \\
Public space, $\mathrm{n}(\%)$ & $410(13.0)$ & $254(11.8)$ & $156(15.4)$ & 0.005 \\
Bystander-initiated CPR, $\mathrm{n}(\%)$ & $1,314(41.6)$ & $898(41.8)$ & $416(41.1)$ & 0.694 \\
Cardiac etiology, $\mathrm{n}(\%)$ & $2,126(67.3)$ & $1,389(64.7)$ & $737(72.8)$ & $<0.001$ \\
VF, $\mathrm{n}(\%)$ & $506(16.0)$ & $301(14.0)$ & $205(20.2)$ & $<0.001$ \\
Endotracheal intubation, $\mathrm{n}(\%)$ & $1,163(36.8)$ & $854(39.8)$ & $309(60.1)$ & $<0.001$ \\
Call to EMS arrival, min, mean (SD) & $6.3(2.6)$ & $6.2(2.5)$ & $6.4(2.7)$ & 0.177 \\
Call to shock by EMS, min, mean (SD) & $9.1(3.3)$ & $9.1(3.2)$ & $9.2(3.4)$ & 0.541 \\
Call to hospital arrival, min, mean (SD) & $30.3(8.8)$ & $28.6(8.6)$ & $33.9(8.4)$ & $<0.001$ \\
Call to intravenous epinephrine administration, & & & $21.3(6.9)$ & \\
min, mean (SD) & & & & \\
\hline
\end{tabular}

CPR, cardiopulmonary resuscitation; VF, ventricular fibrillation; EMS, emergency medical service.

${ }^{*}$ Calculated for cases with VF as initial cardiac rhythm.

field. They are not allowed, however, to administer intratracheal or intraosseous epinephrine nor other drugs such as vasopressin, atropine, and lidocaine. ${ }^{24}$ The local medical protocol directs the ELSTs to administer epinephrine for either shock-resistant VF arrest or witnessed arrest with PEA/asystole under on-line medical direction from the corresponding physician. The protocol allows them to only attempt intravenous access twice, with each attempt lasting no longer than $90 \mathrm{~s}$. The allowable dosage of epinephrine is $1 \mathrm{mg}$ per attempt and they can administer it a total of 3 times every $4 \mathrm{~min}$.

\section{Data Collection and Quality Control}

Data were collected prospectively with the use of a data form based on the Utstein-style international guideline of reporting OHCA. ${ }^{19,20}$ First documented rhythm was recorded and diagnosed on the scene by EMS personnel with semi-automated defibrillators, then confirmed by the physician responsible for on-line medical direction. The times when an EMS call was received and a vehicle arrived at the scene were recorded automatically at the dispatch center. The initiation of bystander CPR was obtained by EMS personnel interviewing bystanders before leaving the scene. The defibrillation time was recorded by a semi-automated defibrillator, and the epinephrine administration time was recorded by the EMS personnel using their watches, which were synchronized with the clock at their dispatch center.

All OHCA survivors were followed for up to 1 month after the event by the EMS personnel and investigators with the cooperation of the Osaka Medical Association and medical institutions in the Osaka area. Neurological outcome was determined by the physician responsible for the care of the patient at 1 month after a successful resuscitation, using the cerebral performance category (CPC) scale: category 1, good cerebral performance; category 2, moderate cerebral disability; category 3 , severe cerebral disability; category 4 , coma or vegetative state; and category 5 , death. ${ }^{25}$

\section{Statistical Analysis}

A primary outcome was regarded as neurologically intact 1month survival as defined by CPC categories 1 or $2 .{ }^{25}$ Secondary outcome measures included return of spontaneous circulation (ROSC) before hospital arrival, ROSC (total), hospital admission, and 1-month survival. The outcomes were com- pared between with and without epinephrine administration, and further compared after stratifying by first documented cardiac rhythm (VF or non-VF). Next, in keeping with our hypothesis that early intravenous epinephrine administration within $10 \mathrm{~min}$ after OHCA occurrence should improve neurological outcome, we subdivided the cases of epinephrine administration into 3 groups: $\geq 10,11-20$ and $\geq 21 \mathrm{~min}$ from the time of the call until epinephrine administration, and compared the patients outcomes with those in the non-epinephrine group.

\section{Statistical Analysis}

Patients' and EMS characteristics and outcomes between groups were compared using a t-test for numerical variables and a chi-square test for categorical variables. Multiple logistic regression analysis assessed the association between the time to epinephrine administration and favorable neurological outcome, and the odds ratios (ORs) and 95\% confidence intervals (CIs) were calculated. As potential confounders, factors that were biologically essential and considered to be associated with clinical outcomes were included in the multivariate analyses: sex, age, location of cardiac arrest, bystander CPR, etiology, first documented cardiac rhythm, endotracheal intubation, and the year of cardiac arrest (time trend). All statistical analyses were performed using SPSS statistical package version 16.0 J (SPSS, Inc, Chicago, IL, USA). All tests were 2 -tailed, and a $\mathrm{P}$ value $<0.05$ was considered statistically significant.

\section{Results}

\section{Overview of OHCA Patients in Osaka}

Figure 1 is an overview of the study patients based on the Utstein template. A total of 9,204 patients resuscitated by epinephrine-capable EMS personnel were registered. Of them, 3,295 were witnessed by bystanders. Among the witnessed cases, 567 had VF including pulseless ventricular tachycardia, and 2,655 PEA/asystole. After excluding 61 shock-responding VF arrests, 3,161 cases were eligible for our analyses. Of these, 1,013 (32.0\%) actually received epinephrine. 
Table 2. Outcomes for OHCA With and Without Epinephrine Administration by Epinephrine-Capable EMS

\begin{tabular}{lccc} 
& No administration & Epinephrine administration & P value \\
n & 2,148 & 1,013 & \\
ROSC before hospital arrival, $\mathrm{n}(\%)$ & $287(13.4 \%)$ & $297(29.3 \%)$ & $<0.001$ \\
Hospital admission, n (\%) & $1,015(47.3 \%)$ & $511(50.4 \%)$ & 0.094 \\
1-month survival, n (\%) & $881(41.0 \%)$ & $432(42.6 \%)$ & 0.385 \\
Neurologically intact 1-month survival, n (\%) & $258(12.0 \%)$ & $137(13.5 \%)$ & 0.245 \\
\hline
\end{tabular}

OHCA, out-of-hospital cardiac arrest; EMS, emergency medical service; ROSC, return of spontaneous circulation.

\begin{tabular}{|c|c|c|c|c|c|c|}
\hline & \multicolumn{3}{|c|}{ VF as initial cardiac rhythm $(n=506)$} & \multicolumn{3}{|c|}{ Non-VF as initial cardiac rhythm $(n=2,655)$} \\
\hline & $\begin{array}{c}\text { No } \\
\text { administration }\end{array}$ & $\begin{array}{c}\text { Epinephrine } \\
\text { administration }\end{array}$ & $P$ value & $\begin{array}{c}\text { No } \\
\text { administration }\end{array}$ & $\begin{array}{l}\text { Epinephrine } \\
\text { administration }\end{array}$ & $P$ value \\
\hline $\mathrm{n}$ & 301 & 205 & & 1,847 & 808 & \\
\hline ROSC before hospital arrival, $\mathrm{n}(\%)$ & $96(31.9 \%)$ & $61(29.8 \%)$ & 0.610 & $191(10.3 \%)$ & $236(29.2 \%)$ & $<0.001$ \\
\hline ROSC (total), n (\%) & $189(62.8 \%)$ & $108(52.7 \%)$ & 0.038 & $827(44.8 \%)$ & $402(49.8 \%)$ & 0.018 \\
\hline Admission, $\mathrm{n}(\%)$ & $177(58.8 \%)$ & $95(46.3 \%)$ & 0.010 & $705(38.2 \%)$ & $336(41.6 \%)$ & 0.097 \\
\hline 1-month survival, n (\%) & $109(36.2 \%)$ & $61(29.8 \%)$ & 0.188 & $150(8.1 \%)$ & $75(9.3 \%)$ & 0.347 \\
\hline Neurologically intact 1-month survival, $\mathrm{n}(\%)$ & $76(25.2 \%)$ & 29 (14.1\%) & 0.006 & $55(3.0 \%)$ & $12(1.5 \%)$ & 0.027 \\
\hline
\end{tabular}

Abbreviations as in Table 2.

\section{Patients' and EMS Characteristics by Epinephrine Administration}

Table 1 shows the characteristics of eligible patients according to epinephrine administration. Mean age was lower in the epinephrine administration group than in the non-administration group, but the male/female ratio was higher in the epinephrine administration group. OHCA among the patients in the epinephrine administration group was more likely to occur in public spaces, be cardiac in etiology and $\mathrm{VF}$, and require endotracheal tube than those in the non-administration group. Although there was no difference between the groups in the time from call to both EMS arrival and defibrillation, the time from call to hospital arrival was significantly longer in the epinephrine administration group than in the non-administration group ( 33.9 vs. $28.6 \mathrm{~min}, \mathrm{P}<0.001$ ). The mean time from call to epinephrine administration was $21.3 \mathrm{~min}$.

\section{Outcomes by Epinephrine Administration}

Table 2 shows the outcomes according to epinephrine administration. Although the rate of ROSC before hospital arrival was significantly higher in the epinephrine administration group than in the non-administration group $(29.3 \%$ vs. $13.4 \%$, $\mathrm{P}<0.001)$, the rate of neurologically intact 1-month survival was lower in them compared with that in the non-epinephrine group ( $4.1 \%$ vs. $6.1 \%, \mathrm{P}=0.028)$. The outcomes of OHCA according to epinephrine administration and the type of first documented cardiac rhythm are listed in Table 3. Although the proportion of ROSC before hospital arrival was significantly higher in the epinephrine administration group than in the non-administration group $(29.2 \%$ vs. $10.3 \%, \mathrm{P}<0.001)$ for non-VF arrests, the epinephrine administration group had a significantly lower rate of neurologically intact 1-month survival than the non-administration group among both $\mathrm{VF}$ ( $14.1 \%$ vs. $25.2 \%, \mathrm{P}=0.006)$ and non-VF arrests $(1.5 \%$ vs. $3.0 \%, \mathrm{P}=0.027)$.

\section{Time of Epinephrine Administration and Favorable Neurological Outcome by the Type of First documented Cardiac Rhythm}

The association between the time from call to epinephrine administration and neurologically intact survival is shown in Figure 2. In VF arrests (Figure 2A), the early group that received epinephrine within $10 \mathrm{~min}$ of the call had a significantly higher rate of neurologically intact 1-month survival compared with the non-epinephrine administration group (66.7\% [6/9] vs. $24.9 \%$ [75/301]; adjusted OR 6.34, [95\%CI 1.49-27.02]), whereas the prolonged epinephrine administration groups (time to epinephrine administration: 11-20 and $\geq 21 \mathrm{~min}$ ) did not. In multivariate analysis, provision of bystander CPR was also associated with better neurological outcome (Figure 3). In non-VF arrests (Figure 2B), however, the proportion of neurologically intact 1-month survival was, however, very low irrespective of the time of epinephrine administration, and there was no significant difference between the groups.

\section{Discussion}

From this prospective registry of OHCA, we have demonstrated that early epinephrine administration within $10 \mathrm{~min}$ of a cardiac arrest increases 1-month survival with favorable neurological outcome after VF arrest, based on our hypothesis. In Japan, specially trained ELSTs are legally allowed to use only intravenous epinephrine on the scene, and the protocol of epinephrine administration is uniform and strict based on the Japan CPR guidelines. ${ }^{23}$ Moreover, all patients enrolled in this study qualified to receive epinephrine. Therefore, we consider that in these situations the possibility of any intentional bias for epinephrine administration would be negligible. Under these uniform conditions, our large-scale population-based observational study covering 8.8 million inhabitants enabled us to evaluate the time-dependent effect of epinephrine on OHCA patients. This is, to our knowledge, the first major 


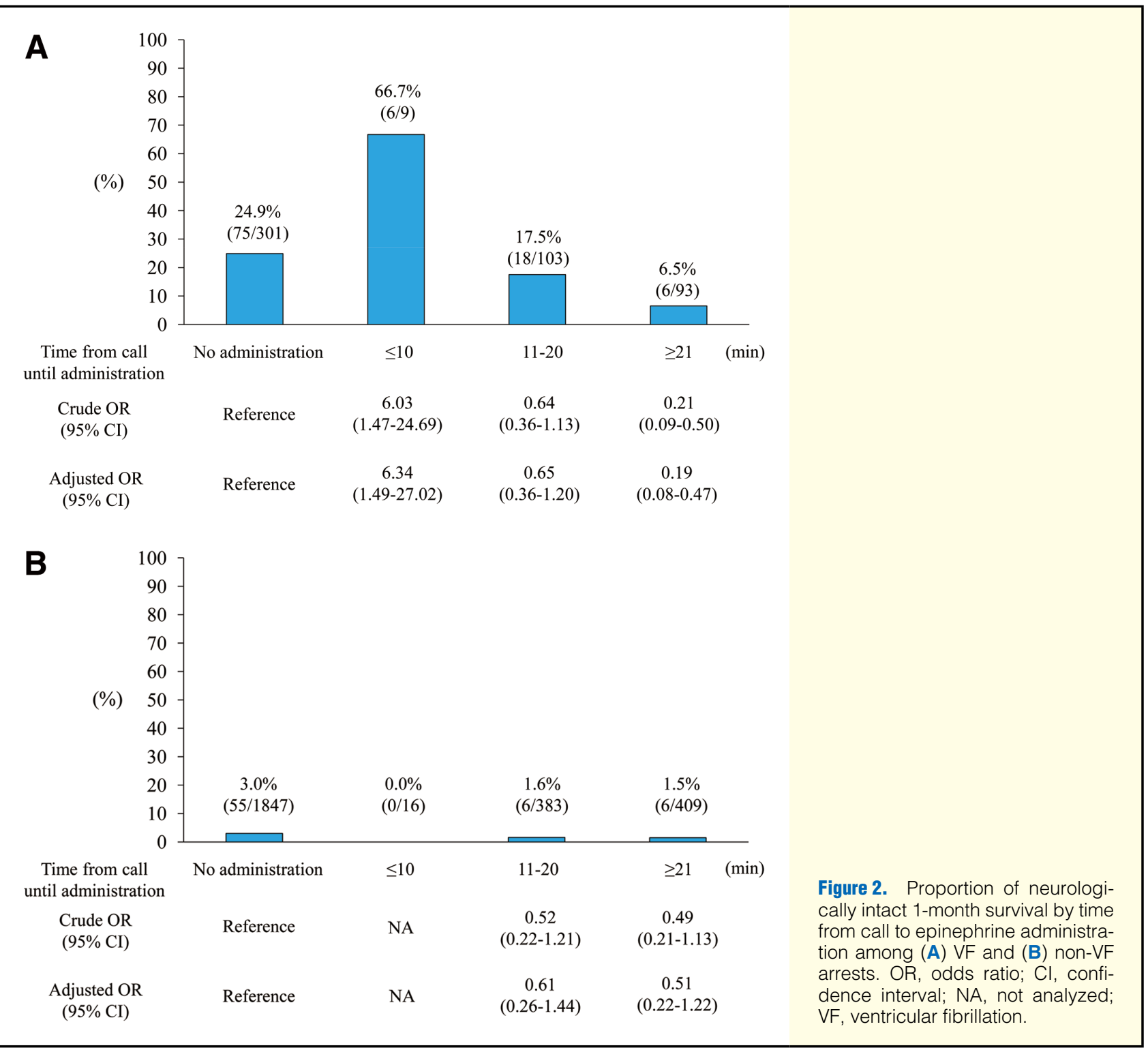

clinical study to show that epinephrine administration can improve survival from OHCA in some selected cases.

Although early epinephrine administration increased 1month survival with favorable neurological outcome after VF arrest, it did not improve neurological outcomes in all cases. These results are consistent with those of some previous observational studies. ${ }^{4-8}$ In Singapore, survival from OHCA showed no change between before and after the introduction of intravenous epinephrine administration to the EMS system. ${ }^{4}$ The Ontario Prehospital Advanced Life Support (OPALS) study, a large "before-and-after" controlled study of the effects of prehospital care, demonstrated that ALS procedures did not produce improved survival from OHCA. ${ }^{8}$ In addition, a large RCT recently failed to show improved survival after drug administration. ${ }^{9}$ However, those studies did not sufficiently consider the time- and cardiac rhythm-dependent effectiveness of epinephrine. Our results showing the effectiveness of early epinephrine administration among VF arrests with no overall improvement suggest that the benefit of epinephrine is different by situation such as the timing of administration and the first cardiac rhythm of OHCA patients.
Our findings that epinephrine has an incremental benefit only when the time to administration was within $10 \mathrm{~min}$ of the cardiac arrest and lower overall survival in the epinephrine administration group were consistent with recent animal studies, ${ }^{10-13}$ in which the time to epinephrine administration was usually within $10 \mathrm{~min}$, compared with $20 \mathrm{~min}$ in human studies, as our data showed. The main pharmacological and physiological effects of epinephrine are to increase aortic diastolic pressure as a result of its alpha-adrenergic agonist properties. This can produce high coronary perfusion pressure, and subsequently, high rates of ROSC and survival. ${ }^{10-13}$ However, epinephrine also increases myocardial oxygen consumption, and postresuscitation myocardial dysfunction. ${ }^{26-28}$ Another animal study indicates that the cardiovascular response to epinephrine varies with the increasing duration of cardiac arrest, and myocardial depression subsequently develops. ${ }^{29}$ These can be possible explanations for the positive results in those with early epinephrine administration and the negative result for overall survival of this study. Although this is the first study to suggest the benefit of early epinephrine administration for OHCA in humans, it is clinically very difficult to 


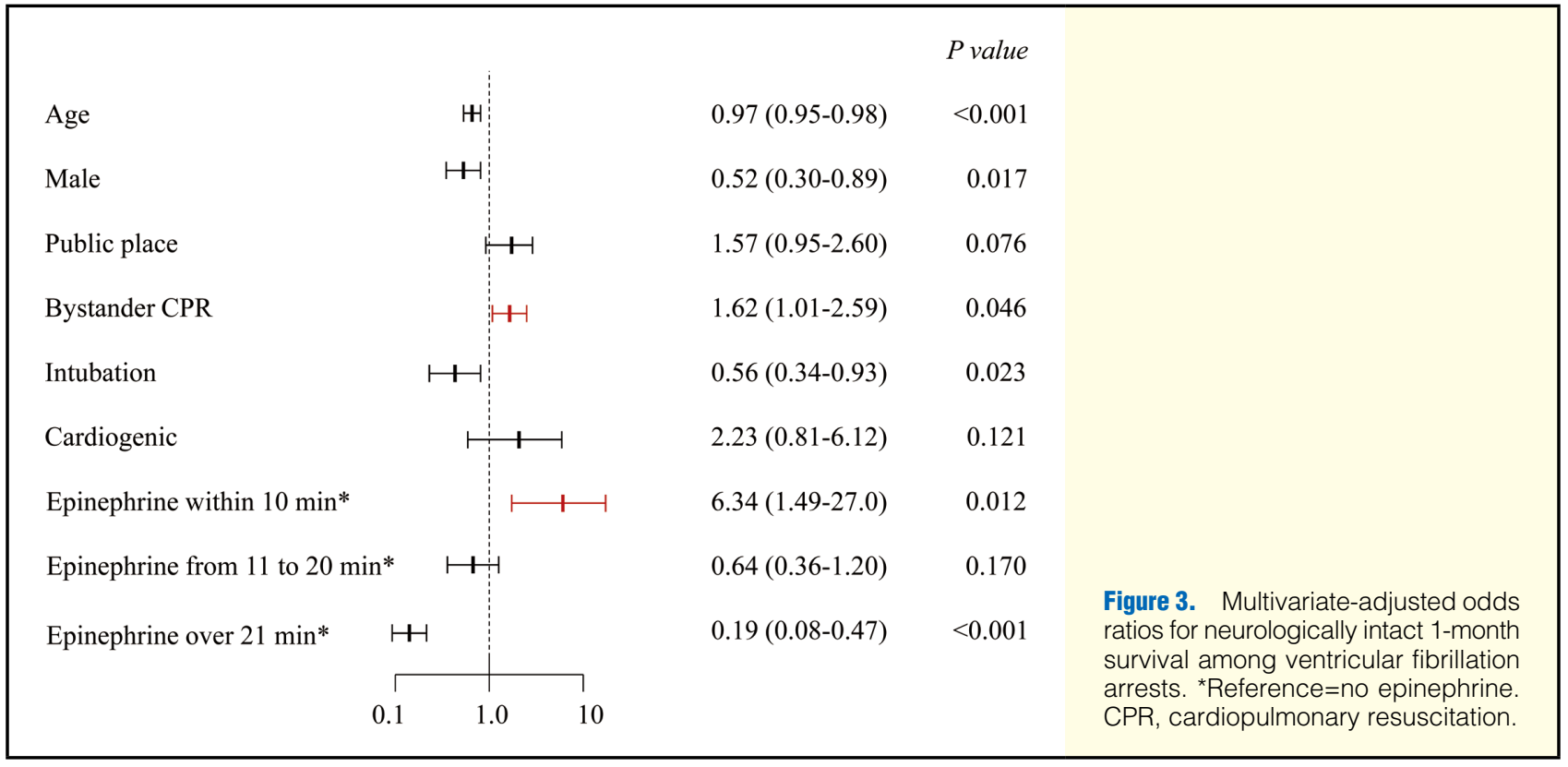

administer epinephrine in this early period and the number of OHCA patients who received intravenous epinephrine within 10 min after OHCA occurrence was small. Further accumulation of patients who have received epinephrine in the early phase after collapse is needed for better ascertainment of the impact of early epinephrine administration on OHCA.

In this study, a time-dependent effectiveness of epinephrine administration was observed only among OHCA patients who had VF as their first documented rhythm. Some studies suggest that survival after epinephrine administration in OHCAs differs by cardiac rhythms. ${ }^{4,9}$ In an $\mathrm{RCT}^{9}$ and a study from Singapore, ${ }^{4}$ the OR of survival in cases of VF arrest was higher compared with non-VF arrests, although the difference was insignificant. Importantly, outcomes were dismal regardless of epinephrine administration among cases of non-VF arrest.

Our data suggest a need to establish a new strategy for the use of epinephrine. Epinephrine use without discrimination should be discontinued, and more targeted use for cardiac arrest patients in the early phase after collapse and those with VF should be considered. In addition, the quality of CPR might have an impact on the effects of epinephrine in OHCA patients. ${ }^{30}$ Early epinephrine administration for VF cardiac arrests following early defibrillation together with continuous high-quality chest compressions should contribute to improving survival. For non-VF cardiac arrests, further efforts including not only ALS procedures but also cause-specific therapy would be needed to increase survival.

Even if early epinephrine administration improved survival from OHCA with VF, it remains difficult to administer epinephrine from intravenous access within $10 \mathrm{~min}$ on the scene. In this study, patients who received intravenous epinephrine within 10 min after the call accounted for only $2.5 \%(25 / 1013)$ of the patients in the epinephrine administration group. Because ELSTs in Japan are only allowed to administer epinephrine via intravenous access, other routes, such as intraosseous access, for administering epinephrine more quickly should be considered. ${ }^{31}$

\section{Study Limitations}

First, as with all observational studies, data integrity, validity, and ascertainment bias are potential limitations. The use of uniform data collection based on the Utstein-style guidelines for reporting cardiac arrest, the large sample size, and a population-based design to cover all known adult cases of OHCA in Osaka were intended to minimize such potential sources of bias. Second, our data do not address potential variability in post-arrest care (hemodynamic support, induced hypothermia, and coronary interventional therapies). ${ }^{32-35}$ Third, there could be unmeasured confounding factors that might have influenced the association between epinephrine administration and outcomes.

Further, large-scale observations or RCT are needed to confirm the results.

\section{Conclusions}

The effectiveness of epinephrine after OHCA depends on the time of administration. When epinephrine is administered in the early phase, it assists in improving the neurological outcome of OHCA with VF. A new strategy using epinephrine for targeted populations with VF cardiac arrests in the early phase after collapse should be considered.

\section{Acknowledgments}

We greatly appreciate the contribution of Hiroshi Morita, Hidekazu Yukioka, Hisashi Ikeuchi, and the other members of the Utstein Osaka Project in the organization, coordination, and oversight of this study as its steering committee. We are also deeply indebted to all of the EMS personnel and concerned physicians in Osaka Prefecture and to the Osaka Medical Association for their indispensable cooperation and support.

\section{Disclosures}

Conflict of Interest: None to declare. Role of Funding Source: This study was supported by a grant for Emergency Management Scientific Research from the Fire and Disaster Management Agency and Foundation from Ambulance Service Department.

\section{References}

1. ECC Committee, Subcommittees, and Task Forces of the American Heart Association. 2005 American Heart Association guidelines for cardiopulmonary resuscitation and emergency cardiovascular care. 
Circulation 2005; 112(Suppl): IV-1-IV-203.

2. Kitamura T, Iwami T, Kawamura T, Nagao K, Tanaka H, Hiraide A; Implementation Working Group for the All-Japan Utstein Registry of the Fire and Disaster Management Agency. Nationwide publicaccess defibrillation in Japan. N Engl J Med 2010; 362: 994-1004.

3. Cummins RO, Ornato JP, Thies WH, Pepe E. Improving survival from sudden cardiac arrest: The "chain of survival" concept. Circulation 1991; 83: $1832-1847$.

4. Ong MEH, Tan EH, Ng FS, Ong ME, Tan EH, Ng FS, et al; Cardiac Arrest and Resuscitation Epidemiology Study Group. Survival outcomes with the introduction of intravenous epinephrine in the management of out-of-hospital cardiac arrest. Ann Emerg Med 2007; 50: 635-642.

5. Holmberg M, Holmberg S, Herlitz J. Low chance of survival among patients requiring adrenaline (epinephrine) or intubation after out-ofhospital cardiac arrest in Sweden. Resuscitation 2002; 54: 37-45.

6. Wang HE, Min A, Hostler D, Chang CC, Callaway CW. Differential effects of out-of-hospital interventions on short- and long-term survival after cardiopulmonary arrest. Resuscitation 2005; 67: 69-74.

7. Ohshige K, Shimazaki S, Hirasawa H, Nakamura M, Kin H, Fujii C, et al. Evaluation of out-of-hospital cardiopulmonary resuscitation with resuscitative drugs: A prospective comparative study in Japan. Resuscitation 2005; 66: 53-61.

8. Stiell IG, Wells GA, Field B, Spaite DW, Nesbitt LP, De Maio VJ, et al. Advanced cardiac life support in out-of-hospital cardiac arrest. N Engl J Med 2004; 351: 647-656.

9. Olasveengen TM, Sunde K, Brunborg C, Throwsen J, Steen PA, Wik L. Intravenous drug administration during out-of-hospital cardiac arrest: A randomized trial. JAMA 2009; 302: 2222-2229.

10. Michael JR, Guerci AD, Koehler RC, Shi AY, Tsitlik J, Chandra N, et al. Mechanisms by which epinephrine augments cerebral and myocardial perfusion during cardio pulmonary resuscitation in dogs. Circulation 1984; 69: 822-835.

11. Otto CW, Yakaitis RW. The role of epinephrine in CPR: A reappraisal. Ann Emerg Med 1984; 13: 840-843.

12. Niemann JT, Cairns CB, Sharma J, Lewis RJ. Treatment of prolonged ventricular fibrillation immediate countershock versus highdose epinephrine and CPR preceding countershock. Circulation 1992; 85: 281-287.

13. Lindberg L, Liao Q, Steen S. The effects of epinephrine/norepinephrine on end-tidal carbon dioxide concentration, coronary perfusion pressure and pulmonary arterial blood flow during cardiopulmonary resuscitation. Resuscitation 2000; 43: 129-140.

14. Reynolds JC, Rittenberger JC, Menegazzi JJ. Drug administration in animal studies of cardiac arrest does not reflect human clinical experience. Resuscitation 2007; 74: 13-26.

15. Rittenberger JC, Bost JE, Menegazzi JJ. Time to give the first medication during resuscitation in out-of-hospital cardiac arrest. Resuscitation 2006; 70: 201-206.

16. Attaran RR, Ewy GA. Epinephrine in resuscitation: Curse or cure. Future Cardiol 2010; 6: 473-482.

17. Iwami T, Nichol G, Hiraide A, Hayashi Y, Nishiuchi T, Kajino K, et al. Continuous improvements in "chain of survival" increased survival after out-of-hospital cardiac arrests: A large-scale populationbased study. Circulation 2009; 119: 728-734.

18. Kitamura T, Iwami T, Nichol G, Nishiuchi T, Hayashi Y, Nishiyama $\mathrm{C}$, et al. Reduction in incidence and fatality of out-of-hospital cardiac arrest in females of the reproductive age. Eur Heart J 2010; 31: $1365-1372$.

19. Cummins RO, Chamberlain DA, Abramson NS, Allen M, Baskett PJ, Becker L, et al. Recommended guidelines for uniform reporting of data from out-of-hospital cardiac arrest: The Utstein Style: A statement for health professionals from a task force of the American Heart Association, the European Resuscitation Council, the Heart and Stroke Foundation of Canada, and the Australian Resuscitation Council. Circulation 1991; 84: 960-975.

20. Jacobs I, Nadkarni V, Bahr J, Berg RA, Billi JE, Bossaert L, et al; International Liaison Committee on Resuscitation; American Heart Association; European Resuscitation Council; Australian Resuscitation Council; New Zealand Resuscitation Council; Heart and Stroke Foundation of Canada; InterAmerican Heart Foundation; Resuscitation Councils of Southern Africa; ILCOR Task Force on Cardiac
Arrest and Cardiopulmonary Resuscitation Outcomes. Cardiac arrest and cardiopulmonary resuscitation outcome reports: Update and simplification of the Utstein templates for resuscitation registries: A statement for healthcare professionals from a task force of the International Liaison Committee on Resuscitation (American Heart Association, European Resuscitation Council, Australian Resuscitation Council, New Zealand Resuscitation Council, Heart and Stroke Foundation of Canada, InterAmerican Heart Foundation, Resuscitation Councils of Southern Africa). Circulation 2004; 110: 3385-3397.

21. International Liaison Committee on Resuscitation. 2005 international consensus on cardiopulmonary resuscitation and emergency cardiovascular care science with treatment recommendations. Circulation 2005; 112: III1 - III136.

22. European Resuscitation Council guidelines for resuscitation 2005. Resuscitation 2005; 67(Suppl 1): S1-S189.

23. Japanese guidelines for emergency care and cardiopulmonary resuscitation. 3rd edn. Tokyo: Health Shuppansha, 2007 (in Japanese)

24. Ambulance Service Planning Office of Fire and Disaster Management Agency of Japan: Effect of first aid for cardiopulmonary arrest (in Japanese). Available at http://www.fdma.go.jp/neuter/topics/ houdou/2212/221203-1 houdou/01-houdoushiryou.pdf (accessed May 2, 2011).

25. Jannett B, Bond M. Assessment of outcome after severe brain damage: A practical scale. Lancet 1975; 1: 480-484.

26. Tang W, Weil MH, Sun S, Noc M, Yang L, Gazmuri RJ. Epinephrine increases the severity of postresuscitation myocardial dysfunction. Circulation 1995; 92: 3089-3093

27. Ditchey RV, Lindenfeld J. Failure of epinephrine to improve the balance between myocardial oxygen supply and demand during closed-chest resuscitation in dogs. Circulation 1988; 78: 382-389.

28. Tovar OH, Jones JL. Epinephrine facilitates cardiac fibrillation by shortening action potential refractoriness. J Mol Cell Cardiol 1997; 29: $1447-1455$.

29. Angelos MG, Butke RL, Panchal AR, Torres CA, Blumberg A, Schneider JE, et al. Cardiovascular response to epinephrine varies with increasing duration of cardiac arrest. Resuscitation 2008; 77: $101-110$.

30. Pytte M, Kramer-Johanse J, Eilevstjønn J, Eriksen M, Strømme TA, Godang K, et al. Haemodynamic effects of adrenaline (epinephrine) depend on chest compression quality during cardiopulmonary resuscitation in pigs. Resuscitation 2006; 71: 369-378.

31. Zuercher M, Kern KB, Indik JH, Loedl M, Hilwig RW, Ummenhofer $\mathrm{W}$, et al. Epinephrine improves 24-hour survival in a swine model of prolonged ventricular fibrillation demonstrating that early intraosseous is superior to delayed intravenous administration. Anesth Analg 2011; 112: 884-890.

32. Neumar RW, Nolan JP, Adrie C, Aibiki M, Berg RA, Bbttiger BW, et al. Post-cardiac arrest syndrome: Epidemiology, pathophysiology, treatment, and prognostication: A Consensus Statement From the International Liaison Committee on Resuscitation (American Heart Association, Australian and New Zealand Council on Resuscitation, European Resuscitation Council, Heart and Stroke Foundation of Canada, InterAmerican Heart Foundation, Resuscitation Council of Asia, and the Resuscitation Council of Southern Africa); the American Heart Association Emergency Cardiovascular Care Committee; the Council on Cardiovascular Surgery and Anesthesia; the Council on Cardiopulmonary, Perioperative, and Critical Care; the Council on Clinical Cardiology; and the Stroke Council. Circulation 2008; 118: 2452-2483.

33. Yokoyama H, Nagao K, Hase M, Tahara Y, Hazui H, Arimoto H, et $\mathrm{al}$; J-PULSE-Hypo Investigators. Impact of therapeutic hypothermia in the treatment of patients with out-of-hospital cardiac arrest from the J-PULSE-HYPO study registry. Circ J 2011; 75: 1063-1070.

34. Yasuda S, Sawano H, Hazui H, Ukai I, Yokoyama H, Ohashi J, et al; J-PULSE Investigators. Report from J-PULSE multicenter registry of patients with shock-resistant out-of-hospital cardiac arrest treated with nifekalant hydrochloride. Circ J 2010; 74: 2308-2313.

35. Nagao K, Kikushima K, Watanabe K, Tachibana E, Tominaga Y, Tada K, et al. Early induction of hypothermia during cardiac arrest improves neurological outcomes in patients with out-of-hospital cardiac arrest who undergo emergency cardiopulmonary bypass and percutaneous coronary intervention. Circ J 2010; 74: 77-85. 